\title{
Occurrence, costs and heritability of delayed selfing in a free-living flatworm
}

\author{
S. A. RAMM*†, D. B. VIZOSO* \& L. SCHÄRER* \\ *Evolutionary Biology, Zoological Institute, University of Basel, Basel, Switzerland \\ $\dagger$ Department of Evolutionary Biology, Bielefeld University, Bielefeld, Germany
}

Keywords:

hypodermic insemination;

inbreeding depression;

Macrostomum hystrix;

mating behaviour;

self-fertilization;

waiting time.

\begin{abstract}
Evolutionary theory predicts that in the absence of outcrossing opportunities, simultaneously hermaphroditic organisms should eventually switch to self-fertilization as a form of reproductive assurance. Here, we report the existence of facultative self-fertilization in the free-living flatworm Macrostomum hystrix, a species in which outcrossing occurs via hypodermic insemination of sperm into the parenchyma of the mating partner. First, we show that isolated individuals significantly delay the onset of reproduction compared with individuals with outcrossing opportunities ('delayed selfing') as predicted by theory. Second, consistent with the idea of $M$. hystrix being a preferential outcrosser under natural conditions, we report likely costs of selfing manifested via reduced hatchling production and offspring survival. Third, we demonstrate that selfing propensity has a genetic basis in this species, with a heritability estimated at $0.43 \pm 0.11$. Variation in selfing propensity could arise due to differing costs of inbreeding among families; despite marked inter-family variation in apparent costs of inbreeding, we found no evidence for such a link. Alternatively, selfing propensity might differ across families because of heritable variation in reproductive traits that determine the likelihood of selfing. We speculate that adaptations to hypodermic insemination under outcrossing, most notably a highly modified copulatory stylet (male copulatory organ) and reduced sperm complexity,

could also facilitate facultative selfing in this species.
\end{abstract}

\section{Introduction}

Self-fertilization provides a viable route to reproduction for many hermaphroditic plants and animals (Jarne $\delta$ Charlesworth, 1993; Goodwillie et al., 2005; Jarne \& Auld, 2006). In principle, individuals reproducing via self-fertilization enjoy a transmission advantage by providing both parental genotypes (Fisher, 1941), but this benefit can be either partially or wholly offset by the negative consequences of inbreeding depression in selfed offspring (Darwin, 1876; Charlesworth \& Charlesworth, 1987). Depending on the relative balance of the costs and benefits, populations are predicted to evolve towards complete outcrossing or complete selfing

Correspondence: Steven A. Ramm, Department of Evolutionary Biology, Bielefeld University, Morgenbreede 45, 33615 Bielefeld, Germany.

Tel.: +49 0521106 2719; fax: +490521 106 6426;

e-mail: steven.ramm@uni-bielefeld.de
(Lande \& Schemske, 1985). Nevertheless, mixed mating strategies are also frequently observed in nature (Jarne \& Charlesworth, 1993; Goodwillie et al., 2005; Jarne \& Auld, 2006). In part, this may reflect variation in ecological factors that influence the costs and benefits of selfing (e.g. Steets et al., 2007; Ellison et al., 2011). Importantly, as mate availability varies in nature, facultative self-fertilization can also represent a conditional strategy that may often pay in the absence of outcrossing opportunities, despite the associated costs, as a form of reproductive assurance (Baker, 1955; Williams, 1975; Tsitrone et al., 2003a).

Recent evolutionary theory has attempted to predict the optimum point at which a simultaneous hermaphrodite should commence reproducing via selfing in the absence of outcrossing opportunities. If selfed offspring suffer significant inbreeding depression, Tsitrone et al. (2003a) predicted that isolated individuals should delay the onset of reproduction beyond the normal age of 
reproductive maturity to maximize the opportunity of encountering a mate (and thereby be able to produce fitter, outcrossed offspring). The optimum length of this delay, termed the 'waiting time', depends on a number of factors including the degree of inbreeding depression and the relative probabilities of encountering a mate vs. survival.

Experimental tests in animals have lent some empirical support to the key prediction that isolated individuals should delay the onset of reproduction via self-fertilization beyond the normal age at first reproduction, especially in Basommatophoran gastropods (review in Escobar et al., 2011). Tsitrone et al. (2003b) reported precisely such a response in the snail Physa acuta, further demonstrating via a full-sib experimental design that this delay to selfing displays significant genetic variation (Tsitrone et al., 2003b; see also Escobar et al., 2007, 2009). Subsequently, a significant delay to commence reproduction has been determined in several additional Basommatophoran species, and in this group, there is a clear interspecific relationship between selfing propensity and inbreeding depression (Escobar et al., 2011). Relatively fewer studies have been conducted in other simultaneously hermaphroditic animals (review in Escobar et al., 2011). In the cestode Schistocephalus solidus, delayed selfing has been reported by one study (Schjørring, 2004), while another study suggested a time cost for outcrossing (Schärer \& Wedekind, 1999). This species is unusual also in that it can exhibit a preference for incestuous mating despite strong inbreeding depression (Schjørring \& Jäger, 2007).

These studies suggest that delayed selfing may be a widespread strategy employed by simultaneous hermaphrodites, but far from all simultaneous hermaphrodites are capable of reproducing via self-fertilization (Jarne $\delta$ Auld, 2006) and even those that are do not necessarily delay reproduction relative to outcrossing (see Escobar et al., 2011). Hence, the taxonomic distribution of delayed selfing remains an important open question. It is also particularly important to investigate the occurrence of selfing in taxa whose reproductive systems would appear to militate against self-fertilization as an option, unlike in plants and gastropods where the often close spatial proximity of the anthers/pistils or the joint male-female organization of the reproductive ducts respectively may often predispose towards selfing in the absence of outcrossing opportunities.

Members of the free-living flatworm genus Macrostomum are highly suitable model organisms to study the reproductive biology of simultaneous hermaphrodites (Schärer \& Ladurner, 2003; Schärer et al., 2011). It was previously assumed that selfing was unlikely in this genus, with Ferguson (1939, p. 144) concluding that 'the mechanism of the hermaphroditic sexual apparatus, which is persistent throughout the genus, is such as to render self-fertilization impossible'. Indeed, there is rel- atively little evidence for selfing among the free-living flatworms in general (von Graff, 1882; Hyman, 1951; Hebert \& Payne, 1985; Peters \& Michiels, 1996; but see Hebert \& Beaton, 1990). However, some Macrostomum species reproduce by hypodermically inseminating sperm into their mating partner, and adaptations to hypodermic insemination could conceivably also facilitate selfing.

Here, we report that one species, Macrostomum hystrix, is indeed capable of reproducing via self-fertilization. We demonstrate that isolated individuals delay reproduction under selfing compared with worms raised with outcrossing opportunities, in line with theoretical predictions (Tsitrone et al., 2003a). Selfing appears to entail a cost, manifested via reduced parental hatchling production and reduced offspring survival. By means of a parent-offspring breeding design, we further show that $M$. hystrix displays genetic variation in its propensity to self. Our results clearly demonstrate that selfing can occur in this free-living flatworm, potentially facilitated by adaptations to hypodermic insemination.

\section{Materials and methods}

\section{Study organism}

Experimental subjects were obtained from a culture of M. hystrix Ørsted 1843 sensu Luther 1905 (see Supplementary Material to Schärer et al., 2011 for further taxonomic details), maintained at the Zoological Institute, University of Basel, from samples originally collected from the San Rossore Regional Park $\left(43.684^{\circ} \mathrm{N}\right.$, $10.283^{\circ} \mathrm{E}$ ) near Pisa, Italy, in May 2010. Worms are maintained at $20{ }^{\circ} \mathrm{C}$ in glass Petri dishes containing $6 \%$ artificial sea water (ASW) and fed with the diatom Nitzschia curvilineata, under conditions that are similar to the ones used to maintain its congener Macrostomum lignano (Schärer \& Ladurner, 2003) and with a generation time of approximately 4 weeks. By closely monitoring experimental subjects in two separate generations, we were able to test for evidence of delayed selfing in $M$. hystrix; evidence for costs of selfing and estimate the heritability of this trait (Fig. 1).

\section{Age at first reproduction of $F_{1}$ individuals (parents)}

To obtain similar-aged hatchlings to use as experimental subjects, 50 adult worms $\left(F_{0}\right)$ were allowed to lay eggs in a Petri dish containing c. $20 \mathrm{~mL}$ of ASW and a dense covering of algae in October 2010. The adults were removed c. $24 \mathrm{~h}$ later, and resulting hatchlings collected after a further 3 days (designated day 0 of the experiment). This effectively prevented any direct contact between the two generations, thus avoiding any possibility for sperm transfer from parents to juveniles, which has previously been observed in another Macrostomum species (D.B. Vizoso, pers. obs.). The med- 
Fig. 1 Experimental design. Hatchling $F_{1}$ worms from an outbred culture $\left(F_{0}\right)$ were allocated to one of two treatments that either did not allow ('Isolated') or did allow ('Triplet') outcrossing opportunities. We tested for evidence of delayed selfing by comparing the age at first reproduction in these two groups of worms (A) and for potential costs of selfing by comparing fecundity within the $F_{1}$ generation (A) and by comparing both survival and fertility between 'Isolated' worms from the $F_{1}$ (outbred) and $F_{2}$ (selfed) generations (B). Finally, to estimate the heritability of age at first reproduction under selfing, we compared the age at first reproduction in $F_{1}$ (parent) and $F_{2}$ (offspring) worms (B). See main text for full explanation.

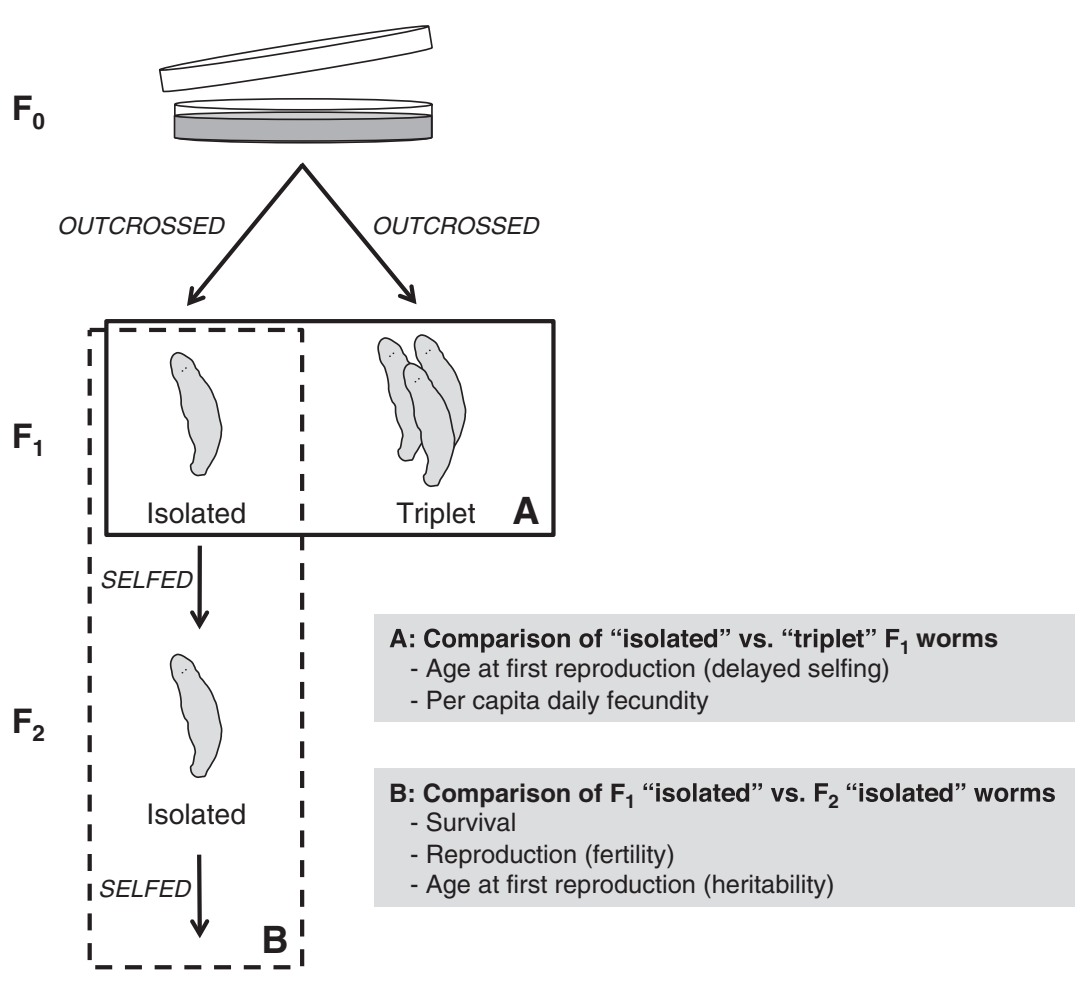

ian time to hatching in M. hystrix under laboratory culture conditions is c. 3 days (S.A. Ramm, unpublished), meaning all $F_{1}$ subjects should have been collected when less than 1 day old. Henceforth, all worms were assumed to be 1 day old at the start of the experiment. In total, $54 F_{1}$ hatchlings were available to use as experimental subjects, of which 24 were randomly allocated to an 'isolated' (selfing) treatment and 30 to a 'triplet' (control) treatment. Isolated worms were transferred individually to 24 wells of a 24-well culture plate, with each well containing $1 \mathrm{~mL}$ of $6 \%$ ASW to which $100 \mu \mathrm{L}$ of a dense algae suspension was added on day 0 of the experiment. Triplets comprised ten groups of three hatchlings per group and were maintained in ten wells of a 12 -well culture plate, with each well containing $3 \mathrm{~mL}$ of $6 \%$ ASW to which $300 \mu \mathrm{L}$ of the same dense algae suspension was added on day 0 of the experiment. Thus, each worm in the experiment was kept at a similar density and had similar food availability, irrespective of treatment group, and all worms were housed within the same plastic box and under identical temperature and lighting conditions. All $F_{1}$ worms remaining in the experiment were transferred to fresh wells prepared in the same way on day 30 of the experiment.

All wells were monitored on days 3, 6, 9, 12, 14, 16 and then (after the first offspring were observed on day 17) daily until day 52 of the experiment to establish the hatching time of all $F_{2}$ offspring in each well. All offspring were removed as soon as they were observed.
Thus, we aimed to remove all offspring within $24 \mathrm{~h}$ of hatching, but the small size and transparency of hatchlings means we cannot completely exclude the possibility that some hatchlings were not removed until the second day on which they were present (once worms have ingested algae they are much easier to identify); henceforth, all $F_{2}$ offspring were assumed to be 1 day old when transferred from the parental wells (see next section).

\section{Age at first reproduction of $F_{2}$ individuals (offspring)}

The first six offspring detected for each isolated parent, assumed to represent up to 24 independent families, were transferred to new, individual wells prepared as for the parents (i.e. with $1 \mathrm{~mL}$ of ASW and $100 \mu \mathrm{L}$ of algae, added in blocks of 24 wells at a time as required, to minimize variation between wells in algae quality). All other hatchlings were removed from the experiment. All $F_{2}$ offspring remaining in the experiment were monitored daily from age of 15 to 50 days, to establish their age at first reproduction. Thus, all $F_{2}$ worms remaining in the experiment were observed every day from day 29 to day 92 of the experiment (except 2 days - 76 and 80 - where observations were not possible for logistical reasons). Again, hatchlings were transferred to fresh wells after $c$. four weeks, with slight variation between individuals due to the differing days on which $F_{2}$ individuals entered the experiment 
(i.e. whole well plates were transferred on the same day). To estimate hatchling survival in a standardized way, we additionally recorded whether or not each $F_{2}$ offspring was still present in the well at age 25 days (i.e. at a time when all worms should be sexually mature).

\section{Statistical analyses}

The 'waiting time' (sensu Tsitrone et al., 2003a) to age at first reproduction under selfing was calculated as the difference in the average time at which $F_{2}$ hatchlings were first observed in the isolated wells, and the average time at which $F_{2}$ hatchlings were first observed in the triplet wells (i.e. assuming reproduction commenced at the same time for all worms within a particular well). For comparison with previous studies (see Escobar et al., 2011), this was assessed using mean values, including a correction for potential bias in the triplet treatment (see Results). The statistical significance of the difference was tested by comparing the median values using a Wilcoxon rank-sum test.

Various potential indicators of the costs of selfing were assessed, to gauge likely 'self-fertilization depression', that is, the combined depression in parental fecundity and offspring fitness under enforced selfing (Jarne et al., 1991; Tsitrone et al., 2003b). First, we compared the per capita daily hatchling production of isolated worms with that of triplet $F_{1}$ worms (once worms had commenced reproduction) using a $t$-test and correcting for unequal variances. Although lower hatchling production could be due to inbreeding depression per se (selfed embryos having higher pre-hatching mortality), it could alternatively represent a reallocation of resources under selfing, resulting in lowered parental fecundity (Tsitrone et al., 2003a). Thus, a reduction in hatchling production does not necessarily imply costs. Next, for each $F_{2}$ family, we compared (i) the proportion of selfed $F_{2}$ offspring surviving to 25 days ('survival') and (ii) of those that survived to 25 days, the proportion that produced at least one hatchling ('reproduction') to the equivalent survival and reproduction probabilities in the $F_{1}$ generation. To take into account error in estimating survival and reproduction in both generations, we employed binomial generalized linear mixed models (GLMMs), using the lme4 package in $\mathrm{R}$, although in the case of reproduction, the estimate of family variation was zero; hence, the analysis is equivalent to a binomial generalized linear model. For each family, the vector of number of successes (surviving/reproducing) and failures (not surviving/not reproducing) within each generation was entered as the dependent variable, the family ID as a random effect and generation $\left(F_{1}, F_{2}\right)$ as a fixed effect. Significance of the 'generation' effect was assessed by comparing models with and without this term, using a likelihood ratio test; a significant genera- tion effect was interpreted as meaning a significant difference in survival/reproduction probability between generations, after controlling for the non-independent sampling of families in each generation.

Note that the selfed $F_{2}$ worms were not directly compared to outcrossed worms, because only the selfed worms were retained in the experiment after the $F_{1}$ generation. Part of any difference between $F_{1}$ and $F_{2}$ worms could therefore be due to environmental factors, although we were careful to maintain conditions across the two generations as closely similar as possible. Our aim was to test whether all three potential indicators of self-fertilization depression changed in the predicted direction in the selfed generation, implying that selfing in $M$. hystrix entails substantial costs.

Heritability of age at first reproduction under selfing was calculated using parent-offspring regression, that is, as the regression coefficient $(b)$ from an ordinary least-squares regression of the mean offspring value for each family on the parental value (Falconer $\&$ Mackay, 1996), based on data from $n=64 F_{2}$ offspring and $n=20 F_{1}$ parents, with $n \leq 6$ offspring per family (mean 3.2 offspring per family, range 1-6). Both unweighted and weighted results to account for differences in family size are reported. Note that the heritability estimate is equivalent to the regression coefficient as in a standard mid-parent analysis, as both maternal and paternal genotypes are transmitted by a single parent, which also means we cannot partition out additive and non-additive sources of variance and our results should be interpreted as providing an upper-limit to the true narrow-sense heritability. Finally, we calculated Pearson correlation coefficients to test for an association across families between selfing propensity (age at first reproduction in the $F_{1}$ generation) and either offspring survival or offspring reproductive capacity (in the $F_{2}$ generation).

\section{Results}

\section{Delayed age at first reproduction under selfing}

Isolated worms forced to reproduce via self-fertilization commenced reproduction substantially later than outcrossing worms kept in triplets (Fig. 2): the mean $( \pm$ S. D.) age at first reproduction in isolated wells was $29.81 \pm 9.13$ days, compared with $19.40 \pm 1.07$ days in triplet wells. Thus, the mean delay is 10.4 days, or $54 \%$, under selfing and $\Delta_{\max }$ (sensu Escobar et al., 2011) is 0.87 . The median ages at first reproduction in isolated and triplet wells were 26 and 19 days, respectively, implying a highly significant delay under selfing (Wilcoxon rank-sum test: $n=31, \quad Z=-3.96$, $P<0.0001)$. There was considerable variation between individuals, with the delay to reproduction ranging from zero (one isolated worm began reproducing on the same day as the first triplet worms) right through to the 


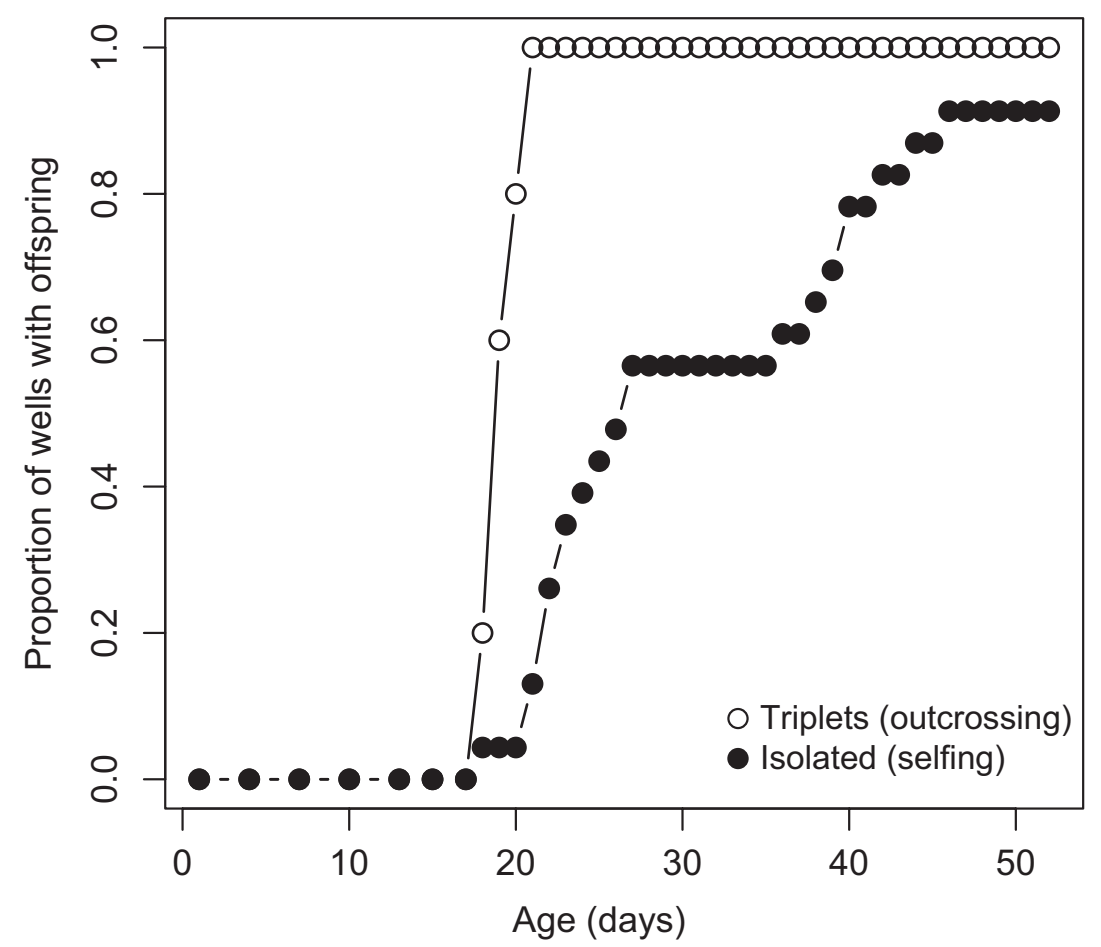

Fig. 2 Delayed selfing in Macrostomum hystrix. The cumulative proportion of experimental groups producing hatchlings as a function of parental age in 'Isolated' worms forced to reproduce via selfing (filled symbols) and 'Triplet' worms with outcrossing opportunities (open circles). Note the substantial delay in the onset of reproduction among isolated individuals. See main text for test statistics.

maximum duration of the experiment (i.e. two surviving worms had not produced any hatchlings when observations in the parental generation ceased on day 52).

Note, however, that one potential problem with this approach is that the age at first reproduction in the 'triplet' treatment may be an underestimation, as it is based on the first hatchlings produced in each well, ignoring any between-individual variation within a well. In practice, individual variation is likely to be small, as we observed very little variation in the estimated age at first reproduction between 'triplet' wells (age range 18-21 days), most wells (80\%) already contained multiple hatchlings on the first or second day on which any hatchlings were observed, and based on the cumulative hatchling production over the course of the experiment, the triplet group of worms appeared to be maximally productive within at most 2 days of the onset of reproduction, with a linear increase in cumulative hatchling production over time thereafter (regression of cumulative hatchling production on age for ages 20-52 days; slope $=35.19 \pm 0.52$ additional hatchlings per day; $\left.r^{2}=0.99, t_{31}=67.94, P<0.0001\right)$. Nevertheless, we deal with the potential bias in two ways:

First, we adopt a more conservative approach for hypothesis testing, by assuming a uniform expected age at first reproduction of 21 days under outcrossing (which is more likely the maximum). This still equates to a statistically highly significant delay under selfing
(Wilcoxon signed-rank test against the null hypothesis of zero delay: $W=88.5$, d.f. $=20, P<0.0001$ ).

Second, to provide an unbiased lower bound for the waiting time (and to facilitate comparison with other study systems), we estimated by simulation the degree to which the age at first reproduction of the outcrossing worms was likely biased downwards by our sampling procedure. To do so, we simulated 1000 random triplet data sets drawn from a standard normal distribution and estimated the systematic bias introduced to the mean and standard deviation of this distribution when only retaining the minimum of the three randomly drawn numbers for each triplet. The resulting sample has a standard deviation of $0.755 \mathrm{~s}$ (where $\mathrm{s}=$ the original standard deviation) and a reduction in mean of $0.826 \mathrm{~s}$. We then assumed a similar bias had been introduced to our real data, and calculated backwards to estimate a corrected mean and standard deviation of the triplet population from which our samples were drawn. This results in an 'unbiased' estimate of age at first reproduction in the triplet treatment of 20.6 days, and a waiting time of $29.8-20.6=9.2$ days (which remains close to the other measured delay).

\section{Costs of selfing}

The potential indicators of self-fertilization depression suggest that the costs of selfing in M. hystrix are likely to 
be substantial. First, we observed a significant reduction in daily hatchling production by 'isolated' compared with 'triplet' worms within the $F_{1}$ generation (mean $\pm \mathrm{SD}$, isolated: $\quad 0.89 \pm 0.42$ hatchlings per day; triplet: $1.15 \pm 0.23$ hatchlings per day; $t$-test assuming unequal variances, $t_{28.47}=2.16, P<0.04$; Fig. 3a). Second, fewer worms survived in the $F_{2}$ generation compared with equivalently treated worms in the $F_{1}$ generation (survival in $F_{1}: 23 / 24$ worms surviving to 25 days $=95.8 \%$; survival in $F_{2}$ : across 21 families, mean $( \pm \mathrm{SE})$ survival $=69.1 \pm 5.8 \%)$, and of those that survived, fewer reproduced in the $F_{2}$ generation compared with equivalently treated worms in the $F_{1}$ generation (proportion of worms surviving to 25 days and producing at least one selfed offspring in $F_{1}: 21 / 23$ worms $=91.3 \%$; across $20 F_{2}$ families, mean proportion reproducing among worms surviving to 25 days $=77.3 \pm 4.4 \%$ ). The statistical significance of these latter two differences was assessed using binomial GLMMs. These analyses indicate that for survival a model containing the 'generation' term provides a significantly better fit than one without (LRT, $\chi^{2}=9.98$, d.f. $=1, P=0.002$ ), with a non-significant trend in the same direction for reproduction (LRT, $\chi^{2}=2.64$, d.f. $=1$, $P=0.10)$. This suggests that the major component of selfing costs in the offspring is probably due to a failure to survive to reproductive age rather than a failure to reproduce if reproductive age is achieved.

\section{Heritable variation in age at first reproduction under selfing}

To determine whether there is a genetic basis to variation among isolated individuals in selfing propensity, we measured the age at first reproduction of up to six offspring from 20 families. In total, $118 F_{2}$ worms were monitored, of which, 64 worms from 20 families had produced offspring by age 50 days (grand mean of family means of age at first reproduction: $29.08 \pm 1.47$ days) and were therefore included in the analysis, by which point we assumed that all worms that would reproduce had in fact already begun to do so. The age at first reproduction did not differ between experimental generations (paired t-test of isolated $F_{1}$ parents vs. isolated $F_{2}$ offspring means: $F_{1}: 29.20 \pm 1.99$; $\left.F_{2}: 29.08 \pm 1.47 ; t_{19}=-0.08, P=0.94\right)$. Based on the ordinary least-squares regression of mean offspring age at first reproduction on parental age at first reproduction, the heritability $\left(h^{2}\right)$ of selfing propensity is $0.47 \pm 0.13$ (Fig. 4, $P<0.003$ ). Alternatively, employing a weighted regression to account for the unequal number of offspring measured per family (Lynch \& Walsh, 1998, p.539), the heritability estimate converged after three iterations to a very similar value of $h^{2}=0.43 \pm 0.11$ (Fig. 4, $P<0.002$ ). These results are qualitatively unchanged if one family with an unusually high value in the offspring generation (based on a single individual) is removed (results not shown).

\section{Does variation in costs predict selfing propensity?}

Finally, we investigated whether variation in other measured traits could explain the across-family variation in selfing propensity. To do so, we tested for an association between the age at first reproduction in each $F_{1}$ parent and the proportion of its $F_{2}$ offspring that survived and reproduced. There was no significant correlation between selfing propensity (age at first reproduction) and either of two proxies for inbreeding depression in the selfed offspring, namely hatchling survival (proportion of hatchlings surviving to age 25 days; $r=0.02, P=0.99$ ) or hatchling reproductive capacity (of those that survived, proportion of hatchlings producing at least one selfed hatchling of their own; $r=0.04, P=0.87)$, despite the fact that both mea-
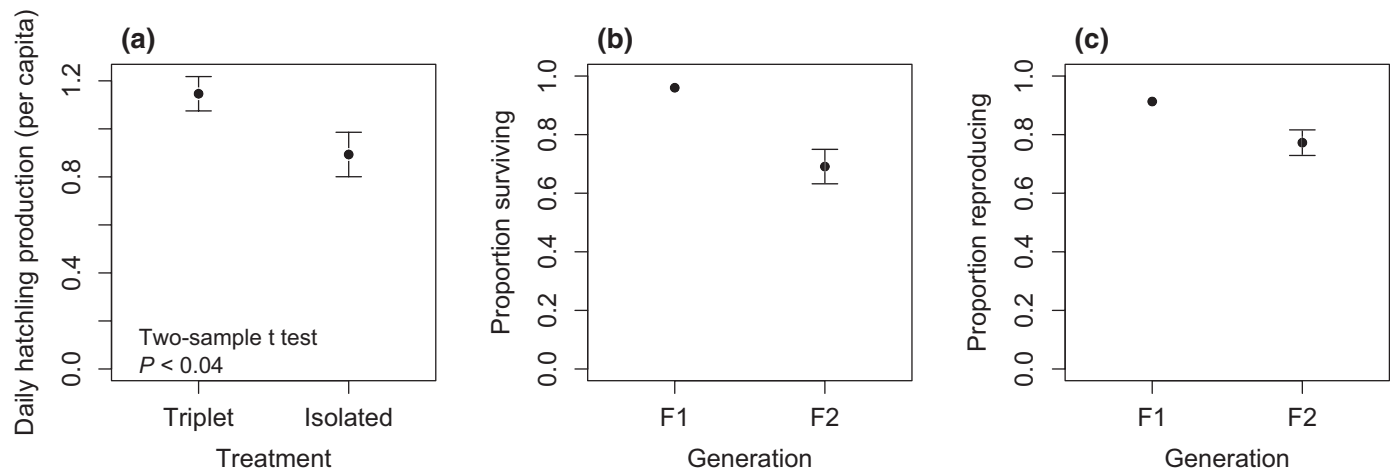

Fig. 3 Costs of selfing in Macrostomum hystrix. (a) Daily hatchling production was lower in isolated compared to triplet worms within the $F_{1}$ generation (mean \pm SEM; $P<0.04$ ). The likely negative impact of selfing was also observed in terms of (b) the proportion of selfed worms surviving to age 25 days and (c) the proportion of selfed worms reproducing, based on comparing isolated $F_{2}$ offspring (selfed worms forced to reproduce by selfing) to their isolated $F_{1}$ parents (outcrossed worms forced to reproduce by selfing). Overall means and standard errors for the $F_{2}$ generation were calculated based on per-family values (see main text for test statistics). 


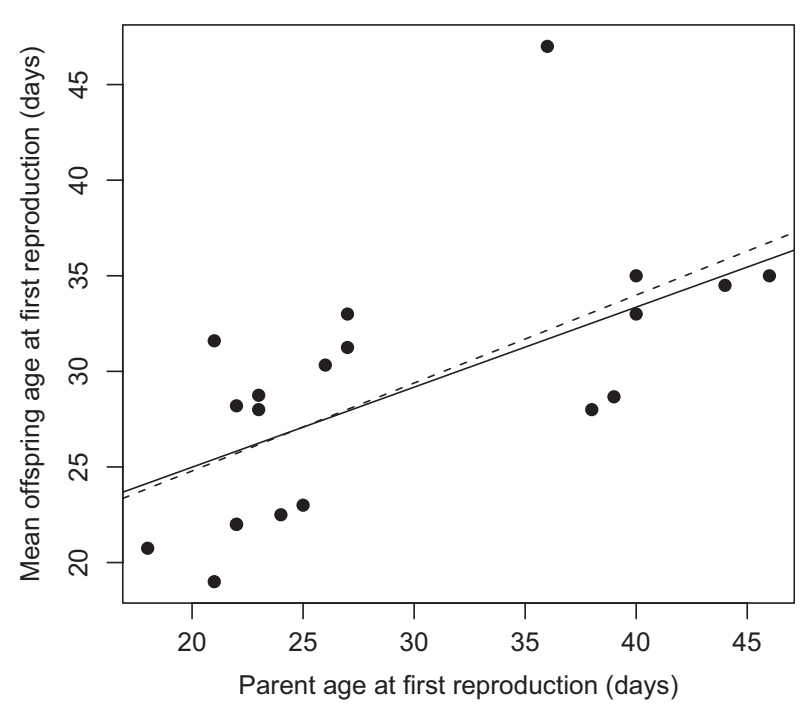

Fig. 4 Heritability of age at first reproduction in Macrostomum hystrix under enforced selfing. Regression of the mean age at first reproduction of offspring in each family on the parental age at first reproduction yields heritability estimates of $0.47 \pm 0.13$ using ordinary least-squares (dotted line, $F_{1,18}=12.31, P<0.003$ ) or $0.43 \pm 0.11$ using weighted regression to account for differences in offspring number per family (solid line, $F_{1,18}=13.90, P<0.002$ ). Note that two overlapping data points occur at $(22,22)$ because two families exhibited identical values in both generations.

sures displayed marked inter-family variation (0$100 \%$ for survival; $33-100 \%$ for reproduction).

\section{Discussion}

\section{Macrostomum hystrix exhibits delayed selfing}

Our study reveals that isolated individuals of the freeliving flatworm $M$. hystrix can reproduce via self-fertilization. However, this species appears to be a preferential outcrosser, as most isolated individuals only begin to self in the extended absence of outcrossing opportunities and at considerable cost in terms of reduced offspring production and fitness. The precise mechanism through which self-fertilization is achieved has not yet been established, but adaptations to hypodermic insemination of another individual may potentially also permit hypodermic self-insemination, which could allow considerable control over the decision to self (see 'Hypodermic Insemination and Self-Fertilization' below).

The significant delay in the onset of reproduction among isolated M. hystrix worms is predicted by theory (Tsitrone et al., 2003a), as inbreeding depression in selfed offspring means it will usually pay for individuals to wait for potential outcrossing opportunities to arise for at least some period beyond sexual maturity, rather than immediately begin reproducing via self-fertilization. Should such outcrossing opportunities fail to materialize, a switch to selfing then provides an alternative route to reproduction, albeit at some cost to offspring fitness (see next section). Similar results to ours have now been obtained in a number of simultaneously hermaphroditic animals, although there has to date been a strong focus on Basommatophoran gastropods (Escobar et al., 2011), but this is to our knowledge only the second report of delayed selfing in the phylum Platyhelminthes (see also Schjørring, 2004; but see Schärer \& Wedekind, 1999), and the first in a nonparasitic flatworm. This suggests that delayed selfing behaviour may be a quite general phenomenon among simultaneously hermaphroditic animals, and indeed analogous phenomena are also observed in hermaphroditic plants under conditions predictive of low outcrossing opportunities (e.g. Vogler et al., 1998; Kalisz et al., 2004).

Delayed selfing represents a conditional reproductive strategy to deal with periodic conditions of low mate abundance, but it also suggests that individuals must often have a reasonable chance of encountering mates, i.e. it is worth waiting for an outcrossing opportunity. A similar conditional strategy to deal with variable mate numbers is found in the outcrossing species $M$. lignano, where individuals show a phenotypically plastic sex allocation in response to different social group sizes, investing more heavily in male reproduction (and at a cost to female reproduction) when they have access to many mates (Schärer \& Ladurner, 2003; Schärer et al., 2005; Janicke \& Schärer, 2009), and becoming most female-biased under isolated conditions (Schärer $\delta$ Janicke, 2009). These two separate phenomena, of facultative selfing and plastic sex allocation, together suggest that dealing with fluctuating environmental conditions may be a problem frequently encountered by this group of flatworms. In the specific case of $M$. hystrix, and although sex allocation has not yet been studied in any detail, it is striking that based on its overall anatomy this species probably exhibits one of the largest testes in the entire genus and can reach very high densities in the field (LS, pers. obs.). These observations suggest $M$. hystrix may in fact frequently encounter environmental conditions of high mate availability and intense sperm competition, coupled with an ability to deal with periods of low mate availability through the delayed selfing behaviour we demonstrate in this study (c.f. for example Lamy et al., 2012).

\section{Selfing is costly}

Delayed selfing is presumed to occur as a form of reproductive assurance. Selfing itself is not automatically favoured because selfed offspring are often less fit than outcrossed offspring due to inbreeding depression (Charlesworth \& Charlesworth, 1987; Charlesworth \& Willis, 2009), and theory predicts that populations will tend to evolve towards two extremes of high selfing 
with low inbreeding depression or low selfing with high inbreeding depression (Lande $\&$ Schemske, 1985; see also Schemske \& Lande, 1985; Goodwillie et al., 2005; Jarne \& Auld, 2006; Escobar et al., 2011). As would be expected for a preferentially outcrossing species we found evidence of substantial costs of selfing in $M$. hystrix, strongly suggesting self-fertilization depression in this species. This conclusion is based on the relatively low survival observed in the $F_{2}$ compared to the $F_{1}$ generation as well as the lower hatchling production of $F_{1}$ worms forced to self. This last result could be due to self-fertilization depression in the wider sense if there is a lower egg-laying rate among selfing worms (Jarne et al., 1991; Tsitrone et al., 2003a), or else to a lower hatching success (presumably due to inbreeding depression causing higher embryo mortality), or both. The past history of inbreeding will strongly affect the expected degree of inbreeding depression (Lande $\delta$ Schemske, 1985). Unfortunately the incidence of selfing in $M$. hystrix under natural conditions is currently unknown, but the availability of a draft genome (http:// www.macgenome.org/) and microsatellite markers (L. Schärer, unpublished) for a congener mean that genetic approaches could feasibly be applied to address this question in the future.

We also found no evidence for a link between selfing propensity and inter-family variation in the costs of selfing based on either hatchling survival or reproductive capacity across families. Such a link could arise through pleiotropy or linkage disequilibrium (Escobar et al., 2009), though our power to detect such a relationship in this experiment was quite low owing to the relatively low level of replication at the individual and family levels. Indeed, previous studies have only convincingly established a link at the intraspecific level in the best studied model to date, $P$. acuta, based on a very large sample of 281 families from 26 populations (Escobar et al., 2009), confirming an earlier observation (Tsitrone et al., 2003b; Escobar et al., 2007). Although there are also clear links between inbreeding depression and selfing propensity at an interspecific level (Husband $\delta$ Schemske, 1996; Goodwillie et al., 2005; Escobar et al., 2011 ), whether or not a particular population exhibits the correlation will depend on its recent selfing history (see Schultz \& Willis, 1995; Escobar et al., 2009). In the case of $M$. hystrix, based on its relatively male-biased sex allocation and evidence of substantial selfing costs, we expect that relatively high density populations (with outcrossing opportunities) may be the norm, but have no direct data to assess whether the absence of correlation between inbreeding depression and selfing propensity is to be expected for our sample.

\section{Delayed selfing exhibits heritable variation}

Employing a quantitative genetic approach, we additionally demonstrate that there is a genetic basis to variation in selfing propensity in $M$. hystrix. We estimated the heritability of age at first reproduction under enforced selfing to be around 0.43 , meaning almost half of the phenotypic variation in the age at first reproduction in isolated offspring was explained by additive or non-additive genetic variation in this trait among the isolated parents. To our knowledge, the heritability of selfing propensity has been investigated previously in only one simultaneously hermaphroditic animal, $P$. acuta, with strikingly similar estimates of 0.43 (Escobar et al., 2007) and 0.46 (Escobar et al., 2009; see also Tsitrone et al., 2003b). Despite the similar outcomes, it should be noted that there are slight differences in experimental design between these studies, in that outcrossing individuals in our experiment had constant access to mates, whereas in the $P$. acuta experiments mate access under outcrossing was only occasional.

Previous studies suggest that selfing propensity in plants also exhibits heritable variation (e.g. Damgaard \& Loeschcke, 1994; Vogler et al., 1998), suggesting the variation we have observed is a very general phenomenon. This heritable variation in selfing propensity could arise either because families vary in the costs of inbreeding depression and thus in the benefits of selfing (but see 'Costs of Selfing' section above), or because of variation in other traits that determine the likelihood of selfing (see 'Hypodermic Insemination and Self-Fertilization' below).

We note that our $h^{2}$ estimate for selfing propensity in $M$. hystrix may actually be somewhat deflated by the absence of $F_{1}$ worms commencing reproduction between day 27 and 34 of the experiment. The most likely explanation for this gap is that it represents an experimental artefact: worms were transferred to fresh wells containing new algae on day 30 of the experiment, and it is likely that immediately prior to this the algae and/or water quality in the original wells had declined to such an extent that any remaining worms that had not yet commenced reproduction were only stimulated to do so by the more propitious conditions when transferred to the new wells (the first offspring appeared 4 days later, and the hatching time in this species is around 3 days; S. A. Ramm, unpublished). This interpretation is also supported by a slight increase in the rate of offspring production among outcrossing individuals shortly after worms were transferred to new wells (data not shown), suggesting that these too may have become limited by algae availability or a declining water quality immediately prior to being transferred.

The substantial genetic variation in selfing propensity we uncovered stands in stark contrast to variation in the age at first reproduction observed among outcrossing individuals. We could not directly estimate the heritability of this trait because we could not identify individuals reproducing in triplets, but in the $F_{1}$ generation it displayed virtually no phenotypic variation 
between experimental replicates (wells). This is in line with previous estimates (e.g. Tsitrone et al., 2003b), and suggests that the variation in delayed selfing behaviour we observed in isolated worms is unlikely to be due to more general variation among worms in the rate at which they become sexually mature.

\section{Hypodermic insemination and self-fertilization}

Outcrossing in $M$. hystrix is achieved by hypodermic insemination, a fertilization route that may be more common in simultaneous hermaphrodites than in gonochorists (Michiels \& Newman, 1998; Michiels \& Koene, 2006; Anthes $\delta$ Michiels, 2007). In this species, sperm possessing a relatively simple morphology compared to other members of the genus are transferred into the parenchyma of the mating partner via a needle-like copulatory stylet (Schärer et al., 2011). As could also be the case in other hypodermically inseminating organisms (see for example Smolensky et al., 2009), we speculate that adaptations to hypodermic insemination - including the needle-like stylet and sperm able to move through the parenchyma - may also facilitate selfing in this species, if the apparatus normally used to stab and inject sperm into the mating partner can be facultatively deployed under isolated conditions to hypodermically self-inseminate sperm. Under this scenario, variation in selfing propensity could then arise due to variation in reproductive traits that are normally expressed under outcrossing but which nevertheless influence the likelihood of self-insemination in the relatively rare instances when low mate availability means that an individual can benefit by switching to selfing. Given the transparent nature of Macrostomum flatworms, $M$. hystrix would appear to represent a highly promising model in which to now investigate patterns of sperm transfer and utilization under both outcrossing and enforced selfing, and to study the traits that influence reproductive success under these two different mating systems. Moreover, the high heritability of the waiting time and short generation time of this species suggest that $M$. hystrix could be a suitable model for studying the experimental evolution of selfing propensity and mating systems.

\section{Conclusions}

We have demonstrated that isolated individuals of the free-living flatworm $M$. hystrix are capable of reproducing via self-fertilization, that they choose to commence doing so only after a substantial delay in their age at first reproduction, that selfing incurs considerable costs and that the propensity to self has a genetic basis in this species. Our study suggests that facultative, delayed selfing may be a widespread phenomenon among simultaneously hermaphroditic animals, potentially in this case facilitated by adaptations to hypodermic insemination.

\section{Acknowledgments}

We thank J. Hottinger, U. Stiefel \& V. Mislin for their technical support, and D. Coltman and two anonymous reviewers for constructive comments on the manuscript, including suggesting the binomial GLMM and unbiased waiting time analyses. This study was funded by a Marie Curie Intra-European Fellowship within the 7th European Union Framework Programme to S.A.R. and a grant from the Swiss National Science Foundation to L.S. (grant number 3100A0127503).

\section{References}

Anthes, N. \& Michiels, N.K. 2007. Precopulatory stabbing, hypodermic injections and unilateral copulations in a hermaphroditic sea slug. Biol. Lett. 3: 121-124.

Baker, H.G. 1955. Self-compatibility and establishment after "long-distance" dispersal. Evolution 9: 347-349.

Charlesworth, D. \& Charlesworth, B. 1987. Inbreeding depression and its evolutionary consequences. Annu. Rev. Ecol. Syst. 18: $237-268$.

Charlesworth, D. \& Willis, J.H. 2009. The genetics of inbreeding depression. Nat. Rev. Genet. 10: 783-796.

Damgaard, C. \& Loeschcke, V. 1994. Genetic variation for selfing rate and the dependence of selfing rate on mating history in Brassica napus (rape seed). Heredity 72: 570-573.

Darwin, C. 1876. The Effects of Cross and Self-Fertilisation in the Vegetable Kingdom. John Murray, London.

Ellison, A., Cable, J. \& Consuegra, S. 2011. Best of both worlds? Association between outcrossing and parasite loads in a selfing fish. Evolution 65: 3021-3026.

Escobar, J.S., Epinat, G., Sarda, V. \& David, P. 2007. No correlation between inbreeding depression and delayed selfing in the freshwater snail Physa acuta. Evolution 61: 2655-2670.

Escobar, J.S., Facon, B., Jarne, P., Goudet, J. \& David, P. 2009. Correlated evolution of mating strategy and inbreeding depression within and among populations of the hermaphroditic snail Physa acuta. Evolution 63: 2790-2804.

Escobar, J.S., Auld, J.R., Correa, A.C., Alonso, J.M., Bony, Y.K., Coutellec, M.-A. et al. 201 1. Patterns of mating system-system evolution in hermaphroditic animals: correlations among selfing rate, inbreeding depression, and the timing of reproduction. Evolution 65: 1233-1253.

Falconer, D.S. \& Mackay, T.F.C. 1996. Introduction to Quantitative Genetics, 4th edn. Longman, Harlow.

Ferguson, F.F. 1939. A monograph of the genus Macrostomum O. Schmidt 1848. Part II. Zool. Anz. 127: 131-144.

Fisher, R. 1941. Average excess and average effect of a gene substitution. Ann. Eugen. 11: 53-63.

Goodwillie, C., Kalisz, S. \& Eckert, C.G. 2005. The evolutionary enigma of mixed mating systems in plants: Occurrence, theoretical explanations, and empirical evidence. Annu. Rev. Ecol. Evol. Syst. 36: 47-79. 
Hebert, P.D.N. \& Beaton, M.J. 1990. Breeding system and genome size of the rhabdocoel turbellarian Mesostoma ehrenbergii. Genome 33: 719-724.

Hebert, P.D.N. \& Payne, W.J. 1985. Genetic variation in populations of the hermaphroditic flatworm Mesostoma lingua (Turbellaria: Rhabdocoela). Biol. Bull. 169: 143-151.

Husband, B. \& Schemske, D. 1996. Evolution of the magnitude and timing of inbreeding depression in plants. Evolution 50: 54-70.

Hyman, L.H. 1951. The Invertebrates: Platyhelminthes and Rhynchocoela: The Acoelomate Bilateria. McGraw-Hill, New York.

Janicke, T. \& Schärer, L. 2009. Sex allocation predicts mating rate in a simultaneous hermaphrodite. Proc. R. Soc. B 276: $4247-4253$

Jarne, P. \& Auld, J.R. 2006. Animals mix it up too: The distribution of self-fertilization among hermaphroditic animals. Evolution 60: 1816-1824.

Jarne, P. \& Charlesworth, D. 1993. The evolution of the selfing rate in functionally hermaphroditic plants and animals. Annu. Rev. Ecol. Syst. 24: 441-466.

Jarne, P., Finot, L., Delay, B. \& Thaler, L. 1991. Self-fertilization versus cross-fertilization in the hermaphroditic freshwater snail Bulinus globosus. Evolution 45: 1136-1146.

Kalisz, S., Vogler, D.W. \& Hanley, K.M. 2004. Context-dependent autonomous self-fertilization yields reproductive assurance and mixed mating. Nature 430: 884-887.

Lamy, T., Lévy, L., Pointier, J.P. \& David, P. 2012. Does life in unstable environments favour facultative selfing? A case study in the freshwater snail Drepanotrema depressissimum (Basommatophora: Planorbidae). Evol. Ecol. 26: 639-655.

Lande, R. \& Schemske, D. 1985. The evolution of self-fertilization and inbreeding depression in plants. I. Genetic models. Evolution 39: 24-40.

Lynch, M. \& Walsh, B. 1998. Genetics and Analysis of Quantitative Traits. Sinauer Associates, Sunderland, MA.

Michiels, N.K. \& Koene, J.M. 2006. Sexual selection favors harmful mating in hermaphrodites more than in gonochorists. Integr. Comp. Biol. 46: 473.

Michiels, N.K. \& Newman, L. 1998. Sex and violence in hermaphrodites. Nature 391: 647.

Peters, A. \& Michiels, N.K. 1996. Evidence for lack of inbreeding avoidance by selective mating in a simultaneous hermaphrodite. Invertebr. Biol. 115: 99-103.

Schärer, L. \& Janicke, T. 2009. Sex allocation and sexual conflict in simultaneously hermaphroditic animals. Biol. Lett. 5: 705-708.

Schärer, L. \& Ladurner, P. 2003. Phenotypically plastic adjustment of sex allocation in a simultaneous hermaphrodite. Proc. R. Soc. B 270: 935-941.
Schärer, L. \& Wedekind, C. 1999. Lifetime reproductive output in a hermaphrodite cestode when reproducing alone or in pairs: a time cost of pairing. Evol. Ecol. 13: 381-394.

Schärer, L., Sandner, P. \& Michiels, N. 2005. Trade-off between male and female allocation in the simultaneously hermaphroditic flatworm Macrostomum sp. J. Evol. Biol. 18: 396-404.

Schärer, L., Littlewood, D.T.J., Waeschenbach, A., Yoshida, W. \& Vizoso, D.B. 2011. Mating behavior and the evolution of sperm design. Proc. Natl Acad. Sci. USA 108: 1490-1495.

Schemske, D. \& Lande, R. 1985. The evolution of self-fertilization and inbreeding depression in plants. II. Empirical observations. Evolution 39: 41-52.

Schjørring, S. 2004. Delayed selfing in relation to the availability of a mating partner in the cestode Schistocephalus solidus. Evolution 58: 2591-2596.

Schjørring, S. \& Jäger, I. 2007. Incestuous mate preference by a simultaneous hermaphrodite with strong inbreeding depression. Evolution 61: 423-430.

Schultz, S. \& Willis, J. 1995. Individual variation in inbreeding depression: the roles of inbreeding history and mutation. Genetics 141: 1209-1223.

Smolensky, N., Romero, M.R. \& Krug, P.J. 2009. Evidence for costs of mating and self-fertilization in a simultaneous hermaphrodite with hypodermic insemination, the Opisthobranch Alderia willowi. Biol. Bull. 216: 188-199.

Steets, J.A., Wolf, D.E., Auld, J.R. \& Ashman, T.-L. 2007. The role of natural enemies in the expression and evolution of mixed mating in hermaphroditic plants and animals. Evolution 61: 2043-2055.

Tsitrone, A., Duperron, S. \& David, P. 2003a. Delayed selfing as an optimal mating strategy in preferentially outcrossing species: theoretical analysis of the optimal age at first reproduction in relation to mate availability. Am. Nat. 162: 318-331.

Tsitrone, A., Jarne, P. \& David, P. 2003b. Delayed selfing and resource reallocations in relation to mate availability in the freshwater snail Physa acuta. Am. Nat. 162: 474-488.

Vogler, D., Das, C. \& Stephenson, A.G. 1998. Phenotypic plasticity in the expression of self-incompatibility in Campanula rapunculoides. Heredity 81: 546-555.

von Graff, L. 1882. Monographie der Turbellarien. I. Rhabdocoelida. Willhelm Engelmann, Leipzig.

Williams, G.C. 1975. Sex and Evolution. Princeton University Press, Princeton, NJ.

Received 4 April 2012; revised 24 August 2012; accepted 28 August 2012 